\title{
Giant Saphenous Vein Graft Pseudoaneurysm and Total Arterial Redo Coronary Revascularization in A High-Risk Patient
}

\author{
Mantov $\mathbf{M}^{1}$, Irqsusi $\mathbf{M}^{1}$, Vondran $\mathbf{M}^{1}$, Andrási TB ${ }^{1^{*}}$, Rastan $\mathbf{A J}^{1}$ \\ ${ }^{1}$ Department of Cardiac and Cardiovascular Surgery, Philipps University of Marburg, Germany
}

"Corresponding author: Andrási TB, Department of Cardiac and Cardiovascular Surgery, Philipps University of Marburg, Baldingerstr, 35033 Marburg, Germany. Tel: +49 6623-5866223; Email: terezia.andrasi@med.uni-marburg.de

Citation: Mantov M, Irqsusi M, Vondran M, Andrási TB, Rastan AJ (2019) Giant Saphenous Vein Graft Pseudoaneurysm and Total Arterial Redo Coronary Revascularization in A High-Risk Patient. Ann Case Report 11: 242. DOI: 10.29011/2574-7754/100242

Received Date: 05 August, 2019; Accepted Date: 03 September, 2019; Published Date: 06 September, 2019

\begin{abstract}
Background: Aneurysmal formations and pseudoaneurysms of venous aortocoronary bypass grafts are rare long term complications. We describe the presumably largest aortocoronary venous graft pseudoaneurym reported till now, which caused global cardiac dysfunction by right ventricular compression and myocardial ischemia due to coronary graft occlusion.

Case Report: A 72-year-old male patient presented with sight of cardiac decompensation 20 years after CABG. CT-scan revealed a giant intrapericardial mass $(17 \times 8 \times 7 \mathrm{~cm})$ causing chronic right dominant cardiac compression and occlusion of all aortocoronary venous grafts. Surgical removal of the pseudoaneurysm and concomitant fully arterial coronary revascularization was performed through re-sternotomy.

Conclusion: Cardiac dysfunction associated to large pseudoaneurysms of coronary grafts is caused by both direct ventricular compression and myocardial ischemia. Therefore, surgical removal of pseudoaneurysms with simultaneous myocardial revascularization remains the treatment of choice for patients with large symptomatic pseudoaneurysms of aortocoronary venous grafts.
\end{abstract}

Keywords: Aortocoronary Venous Bypass; Cardiac Decompensation; Giant Pseudoaneurym; Total Arterial Revascularization

\section{Introduction}

The use of the great saphenous vein was standard of care in the surgical treatment of ischemic heart disease since 1968 [1]. However, aneurysmal dilatations of the Saphenous Vein Grafts (SVG) were firstly reported in 1975 and have been repeatedly documented thereafter [2-12]. These descriptions emphasize it as an unusual but serious complication of Coronary Artery Bypass Graft Surgery (CABG). Treatment remains a challenge regardless of whether performed by interventional means $[6,8-10]$ or open redo surgery [2,6-11].

\section{Case Report}

A 72-year old male patient underwent a triple Coronary Artery Bypass Grafting (CABG) for a 3-vessel-coronary disease after inferior myocardial infarction in 1998 in an external hospital. The left mammary artery was applied to the Left Anterior Descending Artery (LAD), two isolated aortocoronary SVGs were placed to the first diagonal and right posterolateral artery. The patient remained asymptomatic after an uneventful postoperative course. A new significant stenosis of the circumflex artery was treated by coronary stent implantation in 2002 and a small anterior myocardial infarction was treated conservatively in 2003. Progressive right heart symptoms reoccurred in 2012. A CTangiography revealed a dilated, but patent venous graft to the right posterolateral branch was revealed (Figure 1A). Medical treatment was applied. Progressive dyspnea and angina pectoris reoccurred in 2017 , accompanied by severe leg edema. At this time, the patient also suffered from obesity $(171 \mathrm{~kg}, 183 \mathrm{~cm}$, body mass index 51), arterial hypertension, hypolipoproteinemia, hyperuricemia, insulin-dependent diabetes and obstructive sleep apnea. A chest $\mathrm{X}$-ray showed cardiomegaly and mediastinal enlargement (Figure 1B). 


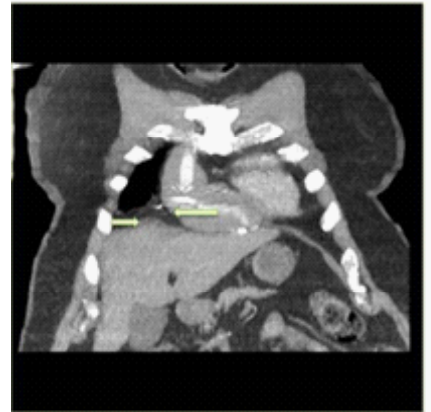

$1 \mathrm{~A}$

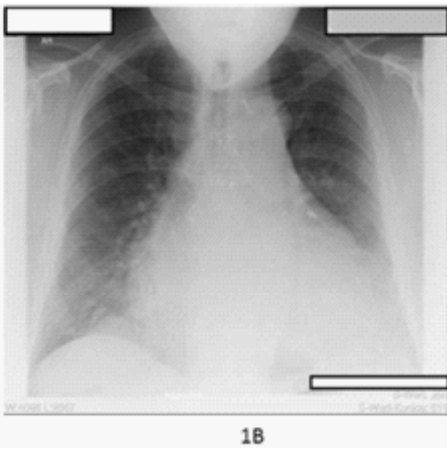

Figure 1: CT-Scan (2012) and X-ray (2017): (A) Computed tomography from the year 2012 showing a partly thrombosed, but still patent pseudoaneurysm (green arrows) of the venous coronary bypass graft to the right territory, (B) chest X-ray on hospital admission in the year 2017.

The coronary angiography revealed proximal occlusion of the Left Ascending Artery (LAD) and the right coronary artery as well as a high-grade stenosis of the obtuse marginal branch (Figure 2A). All bypasses, including the left mammary artery to the LAD and the SVGs were occluded and compressed by the intra pericardial mass (Figure 2B). A new CT-angiography of the chest revealed a giant mass of $17 \times 8 \times 7 \mathrm{~cm}$ size, occupying a significant portion of the anterior mediastinum. The spaceoccupying lesion was not perfused and compromised the function of the right sided cardiac chambers (Figure 3A,3B). The diagnosis of a pseudoaneurysm of the venous graft to right posterolateral branch with massive progression was confirmed. A redo operation was indicated and planned to address the mass removal and repeat coronary revascularization. The preoperative Euro score II was $13.33 \%$.

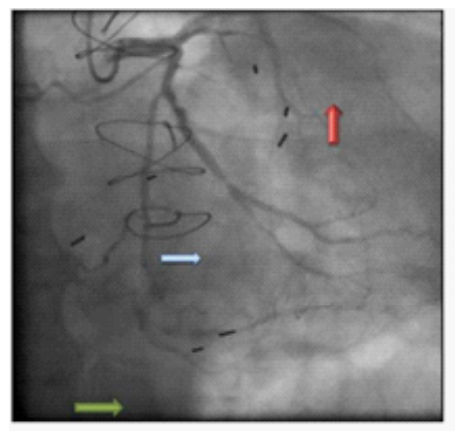

2A

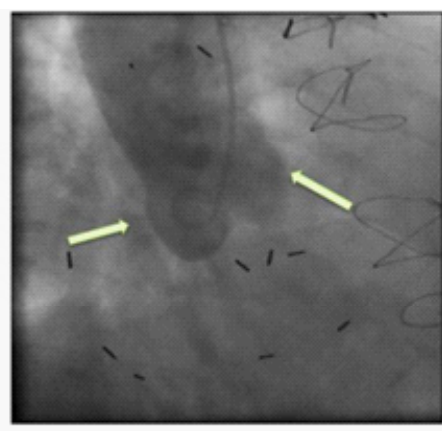

$2 B$
Figure 2: Coronary and aortic root angiogram: (A) Coronary and aortic root angiogram demonstrates occlusion of the left anterior artery (red arrow), the right coronary artery (contrasted by collaterals, green arrow) and a high-grade stenosis of the obtuse marginal branch (blue arrow), (B) lack of patent aortocoronary bypasses.

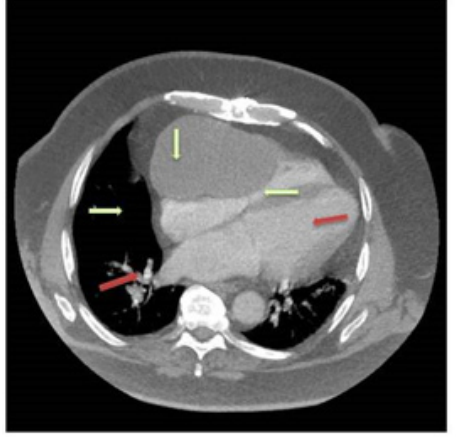

$3 \mathrm{~A}$

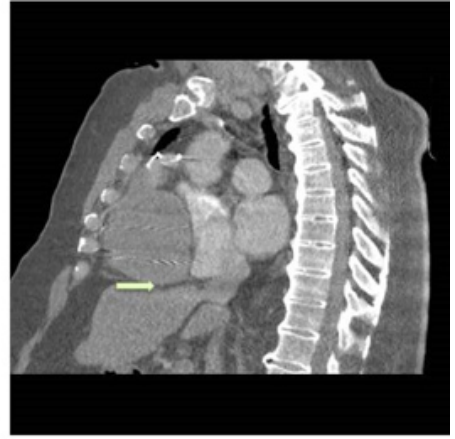

3B
Figure 3: Preoperative CT-scan (2017): (A) CT-Scan form the year 2017 demonstrating a mediastinal mass (green arrows) compressing the right heart chambers, (B) complete occlusion of the venous graft.

Intraoperatively, the giant pseudoaneurysm was identified as a pulseless mass (Figure 4A). The left radial artery and the skeletonized right mammary artery were harvested.Afterconnecting cardiopulmonary bypass in a routine fashion the aneurysm was opened and the massive thrombotic detritus formations were removed (Figure 4B). As expected, no perfusion to the coronary artery was found. Cardioplegic arrest was induced with crystalloid cardioplegia. After complete removal of the giant pseudoaneuysm the native coronary arteries were surgically exposed. The radial artery was anastomosed to the posterior descending artery and was proximally implanted in the ascending aorta. The right mammary artery was anastomosed to the obtuse marginal artery and proximally connected as Y-graft to the radial artery.

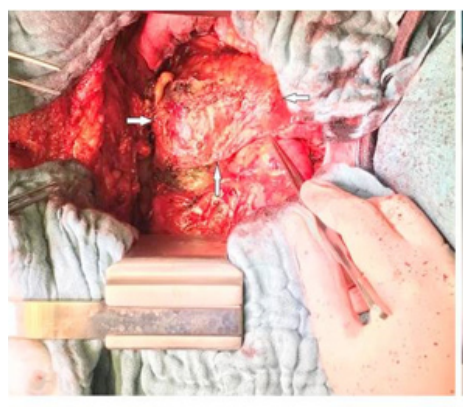

$4 \mathrm{~A}$

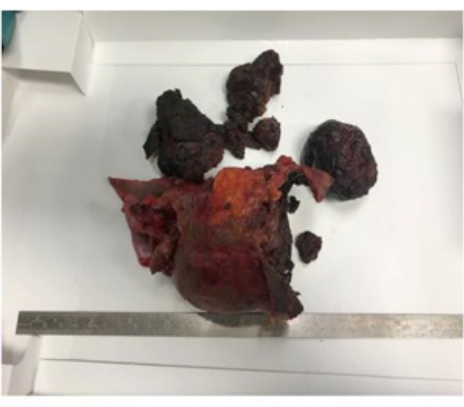

$4 B$
Figure 4: Intraoperative view of the pseudoaneurysm: (A) Intraoperative presentation of the pseudoaneurym, (B) complete removal of the thrombotic mass.

After the uneventful operation, the patient was postoperatively transferred to the intensive care unit under low inotropic support. Two re-operations were bleeding were required on the $1^{\text {st }}$ and $7^{\text {th }}$ postoperative days. Extubation followed within 


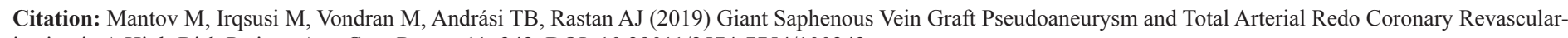
ization in A High-Risk Patient. Ann Case Report 11: 242. DOI: 10.29011/2574-7754/100242

few hours after each operation. The intensive care stay was prolonged by postoperative delirium and respiratory insufficiency. On the 24th postoperative day the patient was transferred to the cardiac rehabilitation clinic and remained symptom-free one year postoperatively. The diagnosis of a venous pseudoaneurysm was histologically confirmed.

\section{Discussion}

The pseudoaneurysmatic degeneration of aortocoronary venous bypass grafts is a rare complication after CABG with an estimated incidence of less than 1\% [2]. Bypass compression associated with stenosis or occlusion results in new ischemic symptoms, whereas a giant intra-pericardial mass may cause right ventricular dysfunction. Previous reports classified venous graft dilatations in true aneurysm (dilatation of an intact venous graft portion) or false aneurysms also known as pseudoaneuryms (extraluminal graft extensions due to disruption of the venous graft wall by trauma, spontaneous rupture or insufficient side branch ligation) $[3,4]$. Pseudoaneurysms can occur early after surgery and are then related to the operative techniques or perioperative factors such as infection; or late after surgery due to small early graft leaks or suture dehiscence [3].

Approximately half of the pseudoaneuryms (47\%) are asymptomatic and present as mediastinal mass on Chest-X-ray [5]. Nonetheless, previous studies revealed that the late occurrence of venous grafts pseudoaneuryms is related to early reoperations after the initial revascularization [2,6]. Even more, the most commonly affected grafts are venous grafts placed on the right coronary territory, followed by venous grafts to the circumflex and LAD arteries. In the literature, the interval from the initial operation to the operative revision of the pseudoaneurysm ranges from 2 to 21 years with a mean interval of 9 years [7]. Therefore, differential diagnosis of a mediastinal mass identified by Chest-X-ray late after CABG surgery should include a possible pseudoaneurym formation.

Computed tomography or magnetic resonance imaging is needed to confirm the diagnosis and give guidance for further therapy [6]. Non-surgical treatment modalities including percutaneous coil embolization, covered stent implantation and Amplatz vascular plug occlusion with low complication and mortality rates [8-10]. Because of the low incidence of large aneurysms there is no consensus on the optimal treatment options. Previous studies concluded that invasive therapy by both, surgical or interventional treatment modalities is indicated when the diameter of the aneurysm exceeded $1 \mathrm{~cm}[7,11]$. The decision to operate or to perform percutaneous closure should be evaluated by the Heart Team based on the complexity of the aneurysm, the devices availability, the lowest risk option and the individual degree of interventional experience.
We believe that interventional pseudoaneurym closure and graft or coronary recanalization is a good treatment for aneurysms that are not causing significant cardiac compression, aneurysms occurring in elderly patients with low life expectancy and patients with high surgical risk [8-10,12-14]. However, the surgical repair should remain the option of choice for all patients with symptoms of right heart compression and indication for repeat coronary revascularization. Because a pseudoaneurysm of an arterial graft has not been reported so far, it seems that total arterial myocardial revascularization might reduce the risk of aneurysm formation and thus, offer a long-time myocardial protection through improved graft patency rates $[15,16]$.

\section{Conclusions}

The present case demonstrates that surgical removal of a giant pseudoaneurysm of a saphenous vein aortocoronary graft and repetitive total arterial coronary revascularizations offers a good outcome, even in a high-risk multimorbide patient suffering from cardiac decompensation.

\section{References}

1. Favaloro RG (1968) Saphenous vein autograft replacement of severe segmental coronary artery occlusion. Ann Thorac Surg 5: 334-339.

2. Smer A, Alla V, Chandraprakasam S, Abuzaid A, Saurav A, et al. (2015) Saphenous venous graft pseudoaneurysm: A Review of the Literature. J Card Surg 30: 70-73.

3. Le Breton H, Pavin D, Langanay T, Roland Y, Leclercq C, et al. (1998) Aneurysms and pseudoaneurysms of saphenous vein coronary artery bypass grafts. Heart 79: 505-508.

4. Kallis P, Keogh BE, Davies MJ (1993) Pseudoaneurysm of aortocoronary vein graft secondary to late venous rupture: Case report and literature review. Br Heart J 70:189-192.

5. Abbasi M, Soltani G, Shomali A, Javan H (2009) A large saphenous vein graft one year after coronary artery bypass graft surgery presenting as a left lung mass. Interact Cardiovasc Thorac Surg 8: 691-693.

6. Ramirez FD, Hibbert B, Simrad T, Pourdjabbar A, Wilson KR, et al. (2012) Natural history and management of aortocoronary saphenous vein graft aneurysms: A systemic review of published cases. Circulation 126: 2248-2256.

7. Aiba M, Anzai T, Kigawa I, Yokokawa H (2015) Large aneurysm in saphenous vein coronary artery graft with fistula: case report and review. Gen Thorac Cardiovasc Surg 63: 293-297.

8. Kim D, Guthaner DF, Wexler L (1983) Transcatheter embolization of a leaking pseudoaneurysm of a saphenous vein aortocoronary bypass graft. Catbet Cardiovasc Diagn 9: 591-594.

9. Barekatain A, Fanari Z, Weiss SA (2015) Aortocoronary saphenous vein graft aneurysm with fistula to the right atrium: percutaneous management of surgical complication. Del Med J 87: 370-375.

10. Dimitri WR, Reid AW, Dunn FG (1992) Leaking false aneurysm of right coronary saphenous vein graft: successful treatment by percutaneous coil embolization. Br Heart J 68: 619-620. 
Citation: Mantov M, Irqsusi M, Vondran M, Andrási TB, Rastan AJ (2019) Giant Saphenous Vein Graft Pseudoaneurysm and Total Arterial Redo Coronary Revascularization in A High-Risk Patient. Ann Case Report 11: 242. DOI: 10.29011/2574-7754/100242

11. Sareyyupoglu B, Schaff HV, Ucar I, Sundt TM, Dearani JA, et al. (2009) Surgical treatment of saphenous vein graft aneurysms after coronary artery revascularisation. Ann Thorac Surg 88: 1801-1805.

12. Krishnasamy P, Gillespie JS, McGlinchey PG, Khan MM, Adgey AA (2007) Giant true saphenous vein graft aneurysm causing cardiac compression: a rare cause of atrial flutter. Int J Cardiol 121: 317-319.

13. Boi A, Sanna F, Rossi A, Loi B (2018) Exclusion of a giant saphenous vein graft pseudo-aneurysm with a "double-layer bridging" technique. Cardiovasc Revasc Med 19: 8-12.
14. Katoh H, Nozue T, Michishita I (2016) A case of giant saphenous vein graft aneurysm successfully treated with catheter intervention. Catheter Cardiovasc Interv 87: 83-89.

15. Gaudino M, Benedetto U, Fremes S, Biondi-Zoccai G, Sedrakyan A, et al. (2018) Radial-Artery or Saphenous-Vein Grafts in Coronary-Artery Bypass Surgery. N Engl J Med 378: 2069-2077.

16. Yanagawa B, Verma S, Mazine A, Tam DY, Jüni P, et al. (2017) Impact of total arterial revascularization on long term survival: A systematic review and meta-analysis of 130,305 patients. Int J Cardiol 233: 29-36. 